\title{
CONSUMERS WITH DIFFERING REACTION SPEEDS, SCALE ADVANTAGES AND INDUSTRY STRUCTURE*
}

\author{
Birger WERNERFELT \\ University of Michigan, Ann Arbor, MI 48109, USA
}

Received March 1983, final version received November 1983

The development of an industry is seen us the outcome of a differential game in which market shares flow in response to price differences. It is hypothesized that while all consumers eventually react to price differences, some are slower than others. If scale advantages have some influence on marginal costs around average market share, then, under reasonable conditions, only $s^{\mathbf{l}}$ ewed size distributions can be locally stable steady states. In addition, and contrary to common belief, the largest firm need not eventually monopolize the industry.

\section{Introduction}

The profit-maximizing price under monopolistic competition is given by the margin' 1 cost plus a mark-up determined by the firm-specific price sensitivity of demand. The simple intuitition exploited in this paper is that if marginal costs decline and firm-specific optimal mark-ups increase with market share, then the two effects could balance each other out and rroducs similar prices for firms of very different sizes.

This situation is found as a locally stable steady state of a non-cooperative differential yame intended to model a mature oligopoly. The model is very simple ant traditional in the sense that each firm uses an open-loop pricing strategy to maximize profits, which are discounted at a common rate. Formally, the open-loop equilibrium concept differs from the closed-loop version in that firms' strategies are allowed to depend on time only. Intuitively, in open-loop models, developments in the state variables cannet lead firms to change plans after these have been nade (that is, a 'plan' is a function of time only). A condition which at least in the medium term can be justified by an adjustment cost argument, as done in Flaherty (1980b). In addition, the short and long run cost function has to satisfy certain technical assumptions, related to increasing returns to scale.

The one novel feature, which together with the increasing returns to scale is responsible for the unusual result, is the dynamic constraint, which

*This paper has benefitted greatly from comments by Hal Varian, David Sappington and two anonymous referees. 
describes the flow of market shares between firms as a function of price differences. Contrary to the ordinary assumption, then, a firm does not lose all of its sales by charging an above-average price for a limited period of time. In particular, it is assumed that different consumers react with differing speed to limited price differences. Intuitively, this will cause a big firm to refrain from trying to capture the last little piece of the market, since doing so would involve extensive and prolonged price cutting on the rest of its already big market share. Conversely, a smail firm can relatively easily take marginal share from a bigger firm. So an asymmetric, non-monopolistic industry structure can be a locally stable steady state. The symmetric solution is unstable under the assumptions of the paper, according to which the cost advantage of a marginally bigger firm dominates the difference in firm-specific price sensitivity of demand, if both firms have approximately average market shares (see fig. 1, notation explained later).

The main contribution of this paper is to offer a deterministic, demandoriented theory of industry structure, which hopefully can complement the stochastic [e.g., Ijiri and Simon (1974), Nelson and Winter (1978)] and costoriented [Flaherty (1980a), Hjalmarsson (1974)] theories in attempts to explain the compelling empirical evidence in the area. [A recent contribution is Buzzell (1980).] Since we shall be dealing with products which in some sense are differentiated, we could also explain skewed size distributions by postulating different cost and demand conditions for different firms. This paper shows that skewed size distributions can be realized even without this. In fact, if firms do face different cost and demand conditions, each of which satisfy the assumptions of this paper, then two types of size distributions are feasible and only the in the some sense 'more' skewed is stable.

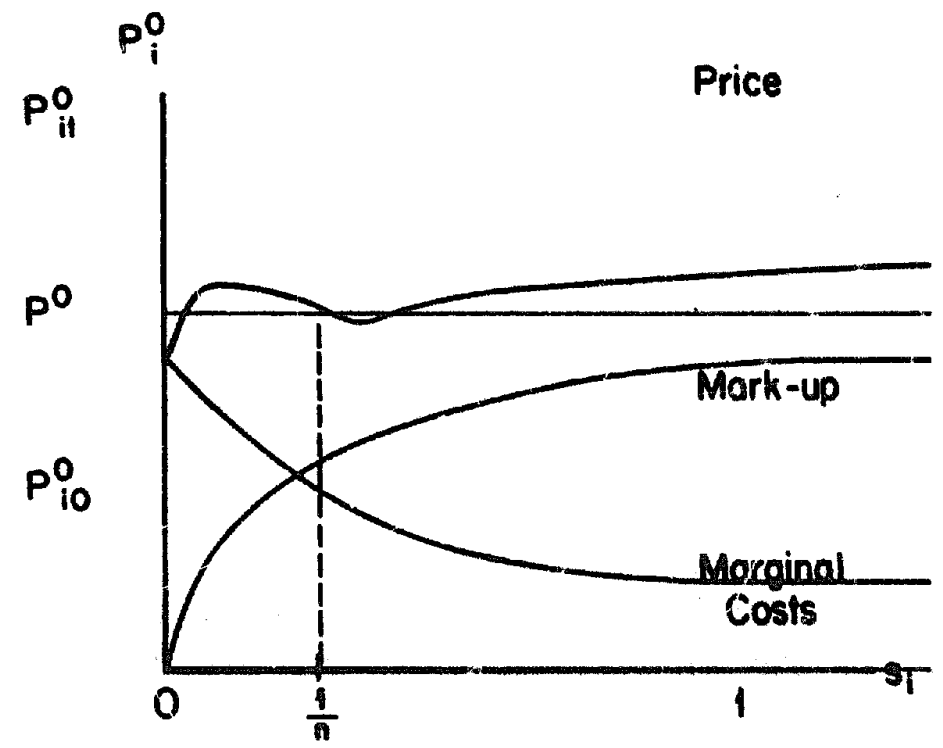

Fig. 1. Steady-state prices as functions of market shares. 
In section 2, I will present and discuss the basic model. The existence and stability properties of the symmetric steady state are established in section 3 , while the asymmetric steady states are investigated in section 4 .

\section{A no-monsense differential game}

In this section, I will present a non-cooperative differential game intended to model the competitive process in a mature oligopoly.

The industry is assumed to consist of $n \geqq 2$ firms, which differ only in their initial market shares, $s_{i 0}>0, i \in\{1, \ldots, n\} \equiv N$. (Of course, $\sum_{i=1}^{n} s_{i 0}=1$.) Each firm selects a pricing strategy, $P_{i}(t)$, from the set of piecewise continuous functions from $[0, \infty)$ to $[0, K]$, where $K$ is some big riumber. In choosing $P_{i}(t)$, firm $i$ will take the strategies of the other firms as given and maximize its infinite horizon profits, discounted at rate $\rho>0$.

If firm $i$ charges the price $p_{i t}$ and has (customer) market share $s_{i t}$, its unit sales volume will be $y\left(p_{i t}\right) s_{i t}$, where $y(x)$ is the product of the number of customers in the market, call $m$, and their identical individual demand functions $y(x) / m$, such that $y(x)$ is total market demand if all firms priced at $x$. It is assumed to be a declining $C^{2}$ function from $[0, K]$ to $[0, \infty)$. Similarly, unit costs will be $C\left(y\left(p_{i t}\right) s_{i t}\right)$, where $C(\cdot)$ is a declining and convex $C^{2}$ function from $[0, \infty)$ to $[0, \infty)$. So the objective of firm $i \in N$ is

$$
\max _{P_{i}(t)} \int_{0}^{\infty} \mathrm{e}^{-\rho t} y\left(p_{i t}\right) s_{i t}\left(p_{i t}-C\left(y\left(p_{i t}\right) s_{i t}\right)\right) \mathrm{d} t
$$

subject to various constraints, in particular the dynamic constraint on market shares. Of course, we need to assume that $y(\cdot)$ and $C(\cdot)$ are such that the maximand is concave in $p_{i t}$. This is the case for all feasible market shares if, for example,

$$
y^{\prime \prime}\left(p_{i t}\right)\left[p_{i t}-C\left(y\left(p_{i t}\right) s_{i t}\right)\right]+2 y^{\prime}\left(p_{i t}\right) \geqq 0,
$$

and

$$
\frac{2}{y\left(p_{i t}\right)}+\frac{y^{\prime \prime}\left(p_{i t}\right)}{y^{\prime}\left(p_{i t}\right)^{2}} \geqq-\frac{C^{\prime \prime}\left(y\left(p_{i t}\right) s_{i t}\right)}{C^{\prime}\left(y\left(p_{i t}\right) s_{i t}\right)}
$$

where primes denote derivatives.

\subsection{The flow of market shares}

While it could be rationalized in many other ways, the dynamic constraint is inspired by the view of consumers developed by Stigler and Becker (1977) and derives from the following intuitive ideas. Adopt the Lancaster view of 
consumption as the production of experiences from products. Suppose now that consumers are uncertain about the degree of differenciation between the products (brands) of diffurent sellers. A first-time buyer, with limited time and information at his disposal, will perform a rough market search and then purchase a brand, which he will learn to use better as he consumes it. When he comes back to the market as a second-time buyer, the brand bought earlier will offer some advantages over other brands, because the user skills have already been acquired. The induced switching cost will vary among consumers and cause some degree of brand loyalty to emerge as the market matures, although some consumers will be more conservative than others. Note that this effect will occur as long as consumers think that products might be heterogeneous, even though in fact they are homogeneous. In order to minimize the number of unusual features in this model, I will here assume that all consumers eventually react to price differences, but that at uny given time some of them take longer than others to do so.

I will write the dynamic constraints as

$$
\dot{s}_{i t}=g\left(s_{i t}, s_{i}^{i}, p_{i t}, p_{i}^{i}\right), \quad i \in N,
$$

where $s_{t}^{i} \equiv\left(s_{i t}, \ldots, s_{i-1 t}, s_{i+1 t}, \ldots, s_{n t}\right)$ and $p_{t}^{i}$ is defined analogously. Since this is a function not usually found in models of demand, we will take some care in explaining the restrictions on it. In addition to some more special conditions to be stated later, the $C^{2}$ function $g(\cdot)$ is assumed to be declining and concave in $p_{i t}$ and $-p_{i}^{i}$. Also, using the shorthand $g_{i}$ for $g\left(s_{i t}, s_{t}^{i}, p_{t s}, p_{t}^{i}\right)$, it follows from the above commentary that $\sum_{i=1}^{n} g_{i}=0$ and that $g(\cdot)=0$ when all prices are identical. If consumers are rational, in the sense that price differences have equal impact, whether they came about through a price increase on the part of one firm or price decreases on the part of all other firms, we have

$$
\frac{\partial g_{i}}{\partial p_{j t}}=-\sum_{k \neq j}^{n} \frac{\partial g_{i}}{\partial p_{k t}}=-\sum_{k \neq i}^{n} \frac{\partial g_{k}}{\partial p_{j t}}, \quad i \in N
$$

The assumption that some consumers are very slow to shift, whereas others are very fast (e.g., because search time has different costs to them) is modeiled as

$$
\frac{1}{s_{i t}} \frac{\partial g_{i}}{\partial p_{i t}} \rightarrow 0 \text { for } \quad s_{i t} \rightarrow 1, \quad i \in N,
$$

and

$$
\frac{1}{s_{i t}} \frac{\partial g_{i}}{\partial p_{i t}} \rightarrow-\infty \text { for } s_{i t} \rightarrow 0, \quad i \in N
$$


According to (B), it is hard to 'get' the last few 'lazy' consumers to buy the product of a quasimonopolist. Conversely, (C) states that a small firm will have an easy time getting the first few aiert buyers. While (B) and (C) may be hard to evaluate in this form, it can be seen. from (6) below, that they are essential for the well-behavedness of the model. Unless (B) holds, a nearmonopolist will charge a very different steady-state price than a monopolist, whereas $(C)$ guarantees that a big number of very small firms will charge steady-state prices 'close to' the competitive price. In other words, the steadystate price function is discontinuous in 1 and 0 , unless (B) and (C) hold. As is the case for most of our other assumptions, (B) and (C) are not necessary, but part of a set of, given the complexity of the model, simple sufficient assumptions. We further assume that

$$
\frac{\partial^{2} g_{i}}{\partial p_{i \imath} \partial s_{j t}}<0, \quad i \neq j, \quad i, j \in N
$$

This says that bigger firms, with more 'fast' customers, loose more by cha ging high prices than smaller firms. Note that our interpretation of $g(\cdot)$ in terms of 'fust' and 'slow' consumers, implies the possibility of a further disaggregation into state variables representing the market shares of a continuum of types of consumers. Since the model already here is very complicated we will look at $g(\cdot)$ as an aggregate description of consumer behavior and will not explicitly model its components. [The function $\dot{s}_{i t}=s_{i t}^{\alpha} \sum_{j \neq i}^{n} s_{j t}^{\alpha}\left(p_{j t}-p_{i:}\right)(0<\alpha<1)$ satisfies all assumptions made on $g_{i}(\cdot)$ alone in this paper.]

\subsection{Necessary conditions for Nash equilibria}

The current-value Hamiltonian for the control problem of the $i$ th firm is given by

$$
\begin{aligned}
& H\left(s_{i t}, s_{i}^{i}, \lambda_{i t}, t ; P_{i}(t), P^{i}(t)\right) \\
& =y\left(p_{i t}\right) s_{i t}\left(p_{i t}-C\left(y\left(p_{i t}\right) s_{i t}\right)\right)+\sum_{j=1}^{n} \lambda_{i j t} g\left(s_{j t}, s_{t}^{j}, p_{j t}, p_{t}^{j}\right),
\end{aligned}
$$

where the costate variables with values $\lambda_{i t} \equiv\left(\lambda_{i 1 i}, \ldots, \lambda_{i n t}\right)$ correspono to the $n$ constraints in $(2)$ and $P^{i}(t)$ denote the strategies of the $(n-1)$ other firms. I W ill now assume the game to be at a Nash equilibrium path and keep the symbols $p_{i t}, p_{t}^{i}, s_{i t}$, and $s_{t}^{i}$ for the values of the corresponding functions along 
this Nash path. ${ }^{1}$ Along this path, the following conditions should hold for all firms:

$$
\begin{aligned}
& s_{i t}\left(y_{i}^{\prime}\left(p_{i t}-C_{i}-s_{i t} y_{i} C_{i}^{\prime}\right)+y_{i}\right)+\sum_{j=1}^{n} \lambda_{i j t} \frac{\partial g_{j}}{\partial p_{i t}}=0, \quad i \in N, \\
& \lambda_{i i t}=\rho \lambda_{i j t}-y_{i}\left(p_{i t}-C_{i}-s_{i t} y_{i} C_{i}^{\prime}\right)-\sum_{j=1}^{n} \lambda_{i j t} \frac{\partial g_{j}}{\partial s_{i t}}, \quad i \in N, \\
& \lambda_{i j t}=\rho \lambda_{i j t}-\sum_{k=1}^{\infty} \lambda_{i k t} \frac{\partial g_{k}}{\partial s_{j t}}, \quad . j \neq i, \quad i, j \in N, \\
& \mathrm{e}^{-\rho t} \lambda_{i j t} s_{j t} \rightarrow 0 \text { for } i t \rightarrow \infty, \quad i, j \in N,
\end{aligned}
$$

where $y_{i}$ and $C_{i}$ denote the values of $y\left(p_{i t}\right)$ and $C\left(y\left(p_{i t}\right) s_{i t}\right)$, and $y_{i}^{\prime}$ and $C_{i}^{\prime}$ denote the derivatives, cvaluated at $p_{i t}, s_{i t}$.

\section{Sieady states}

A steady state of the system represented by eqs. (2) through (5) is characterized by $\dot{s}_{i t}=\lambda_{i j t}=0(i, j \in N)$, and thus by all firms charging constant, identical prices and $\partial \mathrm{g}_{k} / \partial s_{j t}=0(j, k \in N)$. Solving (3) and (4), and dropping time subscripts, we get the steady state price $p_{i}^{0}$,

$$
p_{i}^{0}=C_{i}+s_{i} y_{i} C_{i}^{\prime}-y_{i} /\left(y_{i}^{\prime}+\frac{y_{i}}{\rho} \frac{1}{s_{i}} \frac{\partial g_{i}}{\partial p_{i}}\right), \quad i \in N
$$

This differs from the traditional formula through the second part of the denominator, which corrects for the distributional effects of price competition.

Let us first make some assumptions to guarantee that the dynamical system is well behaved.

By (B) and (6), the steady-state price for $s_{i t} \rightarrow 1$ goes to the monopoly price, given by

$$
p_{i 1}^{0}=C\left(y\left(p_{i 1}^{0}\right)\right)+y\left(p_{i 1}^{0}\right) C^{\prime}\left(y\left(p_{i 1}^{0}\right)\right)-\frac{\dot{y}\left(p_{i 1}^{0}\right)}{y^{\prime}\left(p_{i 1}^{0}\right)}, \quad i \in N,
$$

${ }^{1}$ While it is not the main issue in this paper, a proof of the existence of such a Nash path can be given with a few extra assumptions. In fact, if we construct a discrete version of the game, such that each firm wants to maximize

$$
\sum_{i=1}^{\infty}(1+r)^{-t+1} y\left(p_{i t}\right) g\left(s_{i t-1}, s_{t-1}^{i}, p_{i t-1}, p_{t-1}^{i}\right)\left[p_{i t} C\left(y\left(p_{i t}\right) g\left(s_{i t-1}, s_{t-1}^{t}, p_{i t-1}, p_{t-1}^{i}\right)\right)\right] \text {, }
$$

an open-loop Nash equilibrium exists if the maximand is jointly concave in $p_{i t}$ and $p_{i t-1}$ (rather than in $p_{i}$ alone) and our other assumptions hold [Friedman (1977, Theorem 9.6)]. 
whereas (C) and (6) give the competitive price for $s_{i t} \rightarrow 0$,

$$
p_{i 0}^{0}=C(0), \quad i \in N \text {. }
$$

We also make the natural assumption that the symmetric steady-state price is between the competitive price and the monopoly price, such that

$$
p_{i 0}^{0}<p^{0}<p_{i 1}^{0}, \quad i \in N .
$$

Technically, this amounts to the assumption that economies of scale are not too dramatic cl nse to competition or monopoly. Again here, the model hardly has economic meaning without this assumption. For ease of exposition, we will furthermore assume that

$$
\frac{\partial p_{i}^{0}}{\partial s_{i}}\left(s_{i}\right)=0, \quad i \in N, \quad \text { has at most two solutions in }[0,1]
$$

There is no principal difficulty in analyzing the model without $(F)$ and the same qualitative results could be found, but one could not guarantee the uniqueness of the stable, asymmetric equilibrium. Under these assumptions plus a few more stated in the following, we can sketch the graph of $p_{i}^{0}\left(s_{i}\right)$, depicted in fig. 1 above.

The idea behind this paper is to develop a set of sufficient conditions for this graph to accurately describe $p_{i}^{0}\left(s_{i}^{0}\right)$, for the existence and instability of the middle, symmetric steady state and the existence and stability of the skewed, asymmetric steady state. To prove the instability of the symmetric steady state, we shall need the following assumption:

$$
\begin{aligned}
\left.\left(\frac{\partial p_{i}^{0}}{\partial s_{i t}}-\frac{\partial p_{j}^{0}}{\partial s_{i t}}\right)\right|_{s=1 / n}= & \left(2 y_{i} C_{i}^{\prime}+\frac{1}{n} y_{i}^{2} C_{i}^{\prime}\right. \\
& \left.+\frac{y_{i}^{2}}{\rho} n\left(\frac{\partial^{2} g_{i}}{\partial p_{i} \partial s_{i t}}-\frac{\partial g_{i}}{\partial p_{i}} n-\frac{\partial^{2} g_{i}}{\partial p_{i} \partial s_{j t}}\right) /\left(y_{i}^{\prime}+\frac{y_{i}}{\rho} n \frac{\partial g_{i}}{\partial p_{i}}\right)^{2}\right)\left.\right|_{s=1 / n}<0, \\
i, j \in N . & \text { (G) }
\end{aligned}
$$

The intuition behind $(G)$ is that, in the event of a small increase in $s_{i}$ from $1 / n$, no other firm may react with a larger price cut than firm $i$ itself. $^{2}$ Technically this requires that marginal costs decline rather steeply around average market share, whereas the optimal mark-ups only increase slightly.

${ }^{2}$ One could operate with a threshold market share, if one changed (C) and (G) accordingly and allowed three solutions in $(\mathbb{F})$. 
The assumption will, for example, hold for $y=1, y^{\prime}=1, \rho=1, n=10, C(\cdot)=$ $s_{i i}^{-0.9}$, and $g_{i}(\cdot)=s_{i i}^{0.9} \sum_{j \neq i}^{10} s_{j i}^{0.9}\left(p_{j t}-p_{i t}\right) .(\mathrm{G})$ is by far the strongest and least natural assumption in this paper. Because the cost and demand functions $C(\cdot)$ and $y(\cdot)$ are being supplemented by the demand flow $g(\cdot)$, many of the other assumptions are intransparent, but after closer analysis, quite natural properties of $\boldsymbol{g}(\cdot)$. In this case, however, we have a truly nontrivial property of $g(\cdot)$. To make $(G)$ easier to evaluate we will, therefore, look at an example of $C(\cdot)$ and $y(\cdot)$. In particular, set $C(\cdot)=\left[y\left(p_{i t}\right) s_{i t}\right]^{\beta}$, and $y(\cdot)=p_{i t}^{\gamma},(\beta, \gamma) \in R^{2}$. Given further constraints such that the maximand is concave in $p_{i t},(\mathrm{G})$ reduces to

$$
\left.\left(\left(\frac{\gamma}{p_{i}^{0}}+\frac{n}{\rho} \frac{\partial g_{i}}{\partial p_{i}}\right)^{2} \rho C \beta(\beta+1)-n \frac{\partial g_{i}}{\partial p_{i}}\right)\right|_{s=1 / n}<\left.\left(\frac{\partial^{2} g_{i}}{\partial p_{i} \partial s_{j 1}}-\frac{\partial^{2} g_{i}}{\partial p_{i} \partial s_{i t}}\right)\right|_{s=1 / n} .
$$

So with this choice of $C(\cdot)$ and $y(\cdot), g(\cdot)$ needs the property that price sensitivity increases steeply around average market share, leading to only a small increase in the mark-up. Furthermore, marginal costs should decrease relatively sharply around $1 / n$ as is, e.g., the case for $\beta \sim-\frac{1}{2}$.

To prove the existence of an asymmetric steady state, with, say, $n_{1}$ 'big' and $n_{2}\left(\equiv n-n_{1}\right)$ 'small' firms, we need to assume the existence of a price, such that these market shares will add up to one. If $p_{i}^{0}\left(s_{i}\right)$ has its interior maximum and minimum at $s_{a}$ and $s_{b}$, respectively, we can define

$$
\begin{array}{llll}
\bar{s}^{1}=1 \quad \text { if } \quad p_{i 1}^{0}<p_{i}^{0}\left(s_{a}\right) & \text { or else } & \bar{s}^{1}=\max \left\{s_{i} \mid p_{i}^{0}\left(s_{i}\right)=p_{i}^{0}\left(s_{a}\right)\right\}, \\
\bar{s}_{1}=s_{b} & \text { if } \quad p_{i}^{0}\left(s_{b}\right)>p_{i 0}^{0} & \text { or else } & \bar{s}_{1}=\max \left\{s_{i} \mid p_{i}^{0}\left(s_{i}\right)=p_{i 0}^{0}\right\}, \\
\bar{s}^{2}=s_{a} & \text { if } p_{i}^{0}\left(s_{a}\right)<p_{i 1}^{0} & \text { or else } & \bar{s}^{2}=\min \left\{s_{i} \mid p_{i}^{0}\left(s_{i}\right)=p_{i 1}^{0}\right\}, \\
\bar{s}_{2}=0 & \text { if } \quad p_{i 0}^{0}>p_{i}^{0}\left(s_{b}\right) \text { or else } & \bar{s}_{2}=\min \left\{s_{i} \mid p_{i}^{0}\left(s_{i}\right)=p_{i}^{0}\left(s_{b}\right)\right\},
\end{array}
$$

such that $\bar{s}^{1}, \bar{s}_{1}, \bar{s}^{2}, \bar{s}_{2}$ are the maximal and mininal possible market shares for big and small firns, respectively. So to assure feasibility we need

$$
\begin{aligned}
& n_{1} \bar{s}_{1}+n_{2} \bar{s}_{2}<1, \\
& n_{1} \bar{s}^{1}+n_{2} \bar{s}^{2}>1 .
\end{aligned}
$$

These assumptions again pertain to the relative slopes of the marginal costs and mark-ups, as functions of market share. The meaning of $(\mathrm{H})$ and $(\mathrm{I})$ is, that the negative slope of $p_{i}^{0}$ has to extend over some interval on both sides of $1 / \mathrm{n}$. So the decrease in marginal costs has to dominate the increase 
in mark-up for some range of market share. Without these assumptions, no stable steady state exists.

A final assumption, needed to prove the stability of the asymmetric steady state, is that, relative to the slopes of the steady-state prices, ${ }^{3} \partial g_{i} / \partial p_{k^{-}} i \neq k$ $(i, k \in N)$ is small relative to $-\partial \hat{\partial} g_{i} / \partial p_{i}(i \in N)$. So we need to assume that, at a given asymmetric steady state,

$$
-\sum_{k \neq i, j}^{n} \frac{\partial g_{j}}{\partial p_{k}} \frac{\partial p_{k}^{0}}{\partial s_{i t}}<\frac{\partial g_{j}}{\partial p_{j}} \frac{\partial p_{j}^{0}}{\partial s_{i t}}+\frac{\partial g_{j}}{\partial p_{i}} \frac{\partial p_{i}^{0}}{\partial s_{i t}}, \quad i \neq j, \quad i, j \in N
$$

This assumption does, for example, trivially hold for $n=2$. In general, it seems natural to assume that $s_{i t}$ is affected 'much' more than $s_{k t}$ by a change in $p_{i t}$.

While (A)-(J) seems a formidable set of assumptions, it is.important to note that they are a convenient set of sufficient assumptions for the below results rather than a set of necessary assumptions. Also, not all of them are required for each of our theorems. The assumptions (A)-(E) specify fairly natural properties on $g(\cdot)$, (F) simplifies the treatment by allowing uniqueness, (H) and (I) gives existence and (J) yields stability. The only strong assumption in our main resul below (Theorem 2) is (G). It is hoped that fig. 1 can give some intuitition about the meaning of this very complex simultaneous assumption on three functions.

\section{Results}

Armed with the assumptions above, we can here proceed to demonstrate the results of this paper.

\subsection{Existence of symmetric steady states}

In a symmetric steady state, where each firm has $1 / n$ of the market, (6) reduces to

$$
p^{0}=C\left(\frac{y}{n}\right)+\frac{y}{n} C^{\prime}-\frac{y}{y^{\prime}+\frac{y}{\rho} n \frac{\partial g}{\partial p}},
$$

where $\partial g / \partial p=\partial g_{i} / \partial p_{i \ell}(i \in N)$.

Since the right-hand side of $(7)$ is a continuous function from $[0, K]$ to itself [assume $\left.\left(C_{i}(y / n)+C^{\prime} y / n-y /\left(y^{\prime}+(y / \rho)(\partial g / \partial p) n\right)\right) \in[0, K]\right]$, we arrive, by Brouwer's fixed-point theorem, at:

${ }^{3}$ Unfortunately, the 'e' sy' assumption $\partial^{2} g_{j} / \partial p_{j t} \partial s_{i t}=0(i \neq j, i, j \in N)$ is unrealistic. 
Theorem 1. There exists a symmetric steady-state industry structure.

\subsection{Stability properties of steady states}

In order to investigate the local stability of the system represented by eqs. (2) through (5) in the neighborhood of a steady state, we expand (2), using that $\partial g_{i} / \partial p_{j}=0(i, j \in N)$, at steady states. ${ }^{4}$

$$
\dot{s}_{i t} \simeq \sum_{j=1}^{n} \frac{\partial g_{i}}{\partial s_{j t}}=\sum_{j=1}^{n} \sum_{k=1}^{n} \frac{\partial g_{i}}{\partial p_{k t}} \frac{\partial p_{k}^{0}}{\partial s_{j t}}, \quad i, j \in N
$$

So we want to look at the characteristic roots of the matrix, call $M$, with typical element $\sum_{k=1}^{n}\left(\partial g_{i} / \partial p_{k t}^{0}\right)\left(\partial p_{k z} / \partial s_{j t}\right)(i, j \in N)$.

An important property of $M$ can be brought out by using (A) on a diagonal element,

$$
\begin{aligned}
\frac{\partial g_{i}}{\partial s_{i t}} & =\sum_{j \neq i}^{n} \frac{\partial g_{i}}{\partial p_{j t}} \frac{\partial p_{j}^{0}}{\partial s_{i t}}+\frac{\partial g_{i}}{\partial p_{i t}} \frac{\partial p_{i}}{\partial s_{i t}} \\
& =-\sum_{j \neq i}^{n} \sum_{k \neq i}^{n} \frac{\partial g_{k}}{\partial p_{j t}} \frac{\partial p_{j}^{0}}{\partial s_{i t}}-\sum_{k \neq i}^{n} \frac{\partial g_{k}}{\partial p_{i t}} \frac{\partial p_{i}^{0}}{\partial s_{i t}} \\
& =-\sum_{j \neq i}^{n} \sum_{k \neq i}^{n}\left(\frac{\partial g_{j}}{\partial p_{k t}} \frac{\partial g_{k}^{0}}{\partial s_{i t}}+\frac{\partial g_{j}}{\partial p_{i t}} \frac{\partial p_{i}^{0}}{\partial s_{i t}}\right)=-\sum_{k \neq i}^{n} \frac{\partial g_{j}}{\partial s_{i t}}, \quad i \in N .
\end{aligned}
$$

So all column sums of $M$ are 0 .

The fact that $M$ has less than full rank follows, of course, from the properties of $g(\cdot)$ induced by (A). Since all market shares have to add up 1 then only $(n-1)$ dimensions are free. Accordingly, we shall perforrn our stability analysis in $(n-1)$ dimensions, using the $n$th firm as a buffer to pick up the hypothetical shocks to one of the other market shares.

\subsection{Instability of symmetric steady state}

As a first step towards proving the possibility of this property, note that (D) and (G) imply

\footnotetext{
${ }^{4}$ By operating in $n$ rather than $n-1$ free dimensions, pretending to forget the constraint $\sum_{i=1}^{n} s_{i t}=1$, we can check for the stability against smail unilateral shocks to sales. Working in $n-1$ dimensions, chacking for small shifts in shares, will ease the proof of the analogy of Theorem 3 and complicate that of the analogy of Theorem 4.
} 


$$
\begin{aligned}
\left.\frac{\partial p_{i}}{\partial s_{i}}\right|_{s=1 / n}= & \left(2 y_{i} C_{i}^{\prime}+\frac{1}{n} v_{i}^{2} C_{i}^{\prime \prime}\right. \\
& \left.+\frac{y_{i}^{2}}{\rho} n\left(\frac{\partial^{2} g_{i}}{\partial p_{i} \partial s_{i}}-\frac{\partial g_{i}}{\partial p_{i}} n\right) /\left(y_{i}^{\prime}+\frac{y_{i}}{\rho} n \frac{\partial g_{i}}{\partial p_{i}}\right)^{2}\right)\left.\right|_{s=1 / n} ^{<0, \quad i \in N,}
\end{aligned}
$$

where $s=1 / n$ is used to denote that all derivatives are evaluated at the symmetric steady state. So a (hypothetical) small unilateral increase in $s_{i}$, from the symmetric steady state, will cause firm $i$, ceteris paribus, to want to lower its price. The mechanism underlying this phenomenon is again that the resulting decline in marginal costs will dominate the decrease in the firmspecific price sensitivity of demand. This is a likely outcome if marginal cost decline steeply around average firm size, a typical phenomenon in young, pre 'shakeout' industries with 'too many' contestants.

At the symmetric steady state, $\partial g_{i} / \partial s_{j t}=\partial g_{k} / \partial s_{l t}(i \neq j)$ when and only when $k \neq l(i, j, k, l \in N)$, such that $M$ is symmetric with identical off-diagonal elements. Any $(n-1) \times(n-1)$ matrix, formed by removing one row and the corresponding column from $M$, will, of course, have the same properties. Therefore,

$$
\left.\left(\frac{\partial g_{i}}{\partial s_{i t}}-\frac{\partial g_{i}}{\partial s_{j t}}\right)\right|_{s=1 / n}=\left.\left.\left(\frac{\partial g_{i}}{\partial p_{i t}}-\frac{\partial g_{i}}{\partial p_{j t}}\right)\right|_{s=1 / n}\left(\frac{\partial p_{i}^{0}}{\partial s_{i t}}-\frac{\partial p_{j}^{0}}{\partial s_{i t}}\right)\right|_{s=1 / n}, \quad i, j \in N
$$

is a root of any such $(n-1) \times(n-1)$ matrix. By $(G)$, this root is positive.

We thus have:

Theorem 2. Under $(A),(D)$ and $(G)$ the symmetric steady state is locally unstable.

\subsection{Existence of asymmetric stealy states}

We can here prove the existence of two asymmetric steady states by the Poincare-Hopf theorem. The theorem uses the Poincare index, which is based on the sign of the rleterminant of the negative of the linearized system at an equilibrium of a djnamical system. According to the theorem, in a well-behaved dynamical system, the number of positive such determinants exceeds the number of negative ones by 1 [see, e.g., Varian (1981, sect. 2.3)]. Since $M$ had all positive eigenvalues at the symmetric steady state, the relevant sign at that equilibrium is negative and we thus have two more steady states. 30 : 
Theorem. 3. Under $(A)-(I)$, there exists an asymmetric steady-state industry structure with $n_{1}$ big and $n_{2}$ small firms. ${ }^{5}$

\subsection{Stability of asymmetric steady states}

To analyze this case, note that the derivative below,

$$
\frac{\partial p_{j}^{0}}{\partial s_{i t}}=\frac{y_{j}^{2}}{\rho} \frac{1}{s_{j t}} \frac{\partial^{2} g_{j}}{\partial p_{j} \partial s_{i t}} /\left(y_{j}^{\prime}+\frac{y_{j}}{\rho} \frac{1}{s_{j t}} \frac{\partial g_{j}}{\partial p_{j}}\right)^{2}, \quad i \neq j, \quad i, j \in N,
$$

is negative by (D).

So all diagonal elements of $M$,

$$
\frac{\partial g_{i}}{\partial s_{i t}}=\sum_{j \neq i}^{n} \frac{\partial g_{i}}{\partial p_{j}} \frac{\partial p_{j}}{\partial s_{i t}}+\frac{\partial g_{i}}{\partial p_{i}} \frac{\partial p_{i}}{\partial s_{i t}}, \quad i \in N
$$

are negative at asymmetric steady states, since these also have the property that $\partial p_{i} / \partial s_{i t}>0(i \in N)$. Also, by $(J)$, all off-diagonal elements of $M$ are positive.

Thus, under (A), (D) and (J), all column sums of $M$ are zero, all diagonal elements are negative, and all off-diagonal elements are positive. Therefore, any $(n-1) \times(n-1)$ matrix, formed by removing one row and corresponding column from $M$, will be a Hadamard matrix with negative diagonal. All eigenvalues of these matrices will thus have negative real parts [see, e.g., Murata (1977, ch. 1, Theorem 20)]. We then have:

Theorem 4. Under $(A),(D)$ and $(J)$, asymmetric steiddy states are locally stable.

\section{Conclusion}

The paper has demonstrated that a combination of increasing returns to scale and brand loyalty can lead to stable asymmetric, but unstable symmetric open-loop equilibria. While one must expect these results to generalize to ordinary closed-loop equilibria, it is not clear how price functions which are not piecewise continuous will affect them. Since the dynamic framework in this paper is unusual in economics, it is hard to evaluate the strength of the assumptions made on the market share flow function. As more models of this type work their way into the literature, it will hopefully be easier to see just how special the case described here is.

${ }^{3}$ A closed-loop model might maike it defensible to assume more than the two solutions in (F) and thus allow explanation of a more complex inclustry structure. 
Conversely, the results here might facilitate interpretation of other dynamic models of demand.

For purposes of explaining the common occurence of skewed size distributions, the model in this paper gives just one, probably not very important, mechanism which can generate the observed phenomena. In practice, market segmentation might play a far greater role than the cost and demand curve properties studied here. Preliminary cross section studies do, however, seem to indicate that average (across several selected industries) realized mark-ups and costs exhibit the market share dependence described in fig. 1. (This is of course a very incomplete test.)

A theoretical drawback of infinite-horizon differential games is that neither existence (see, however, footnote 1) nor uniqueness results are available for such models. An advantage, however, is that economies of scale and demand rigidities can be treated more readily in the dynamic framework.

On the empirical side, a problem with the particular model analyzed here is that it seems unrealistic to argue for more than two firm sizes in the deterministic open-loop format used. As menioned in footnote 5, however, it is possible that a closed-loop format could overcome this problem. An attractive feature of the model is that the unit mark-up grows with market share with the result that the well-known relation between market share and profitability [see, e.g., Buzzell et al. (1975)] is refound.

If the mechanisms of the present model work to turn a small difference in market share into a bigger one, compounded by a mark-up difference, there are interesting strategic implications for the involved firms. Assuming that the small firm decides against trying to fight the odds, the natural response is to try to segment the market in such a way that it can sustain a larger equilibrium market share. Conversely, the bugger firms will try to prevent or pre-empt this segmentation. This, together with the equilibrating mechanisms described here, will tend to shift competitive emphasis from price to advertising.

The major public policy implications of the model would seem to follow from the result that economies of scale do not nesessarily lead to monopoly.

\section{References}

Buzzell, R.D., 1980, Are there natural market structures?, Mimeo. (Graduate School of Business Administration, Harvard University, Cambridge, MA).

Buzzell, R.D. B.T. Gale and R.G.M. Sultan, 1975, Market share - A key to profitability, Harvard Business Review 53, no. 1, 97-106.

Flaherty, M.-T., 1980a, In fustry structure and cost reducing investment, Econometrica 48, 11871209.

Flaherty, M.-T., 1980b, Dynamic limit pricing, barriers to entry and rational firms, Journal of Economic Theory 23, 160-182.

Friedman, J.W., 1977, Oligopoly and the theory of games (North-Holland, Amsterdam).

Hjalmarsson, L., 1974, The size distribution of establishments and firms derived from an optima! process of capacity expansion, European Economic Review 5, 123-140. 
Ijiri, Y. ad H.A. Sinon, 1974, Interpretations of departures from the Pareto curve firm-size distribution, Journal of Political Economy 82, 315-331.

Murata, Y., 1977, Mathematics for stability and optimalization of economic systems (Academic Press, New York).

Stigler, G.J. and G.S. Becker, 1977, De gustibus non est disputantum, American Economic Review 67, 76-90.

Nelson, R.R. and S.G. Winter, 1978, Foross generating and limiting competition under Schumpeterian competition, Bell Iournal of Economics 9, 524-548.

Varian, H.R, 1981, Dynamical systems with applications to economics, in: K.J. Arrow and M.P. Intriligator, eds., Handbook of Mathematical Economics, Vol. 1 (North-Holland, Amsterdam) 93-110. 